\title{
Using a hepatitis B surveillance system evaluation in Fujian, Hainan, and Gansu provinces to improve data quality and assess program effectiveness, China, 2015
}

Hui Zheng ${ }^{1}$, Alexander J. Millman², Jeanette J. Rainey ${ }^{3}$, Fuzhen Wang ${ }^{1}$, Rui Zhang ${ }^{4}$, Hong Chen ${ }^{4}$, Zundong Yin ${ }^{1}$, Huaqing Wang ${ }^{1}$ and Guomin Zhang ${ }^{1 *}$ (D)

\begin{abstract}
Background: Monitoring hepatitis B surveillance data is important for evaluating progress towards global hepatitis B elimination goals. Accurate classification of acute and chronic hepatitis infections is essential for assessing program effectiveness.

Methods: We evaluated hepatitis B case-reporting at six hospitals in Fujian, Hainan and Gansu provinces in 2015 to assess the accuracy of case classification. We linked National Notifiable Disease Reporting System (NNDRS) HBV case-reports with hospital information systems and extracted information on age, gender, admission ward and viral hepatitis diagnosis from medical records. To assess accuracy, we compared NNDRS reported case-classifications with the national HBV case definitions. Multivariable logistic regression was used to identify factors associated with misclassification.

Results: Of the 1420 HBV cases reported to NNDRS, 23 (6.5\%) of the 352 acute reports and 648 (60.7\%) of the 1068 chronic reports were correctly classified. Of the remaining, 318 (22.4\%) were misclassified and 431 (30.4\%) could not be classified due to the lack of supporting information. Based on the multivariable analysis, HBV cases reported from Hainan ( $(\mathrm{aOR}=1.8 ; 95 \% \mathrm{Cl}: 1.3-2.4)$ and $\mathrm{Gansu}(\mathrm{aOR}=12.7 ; 95 \% \mathrm{Cl}: 7.7-20.1)$ along with reports from grade 2 hospitals (aOR $=1.6 ; 95 \% \mathrm{Cl}: 1.2-2.2$ ) and those from non-HBV related departments ( $\mathrm{aOR}=5.3 ; 95 \% \mathrm{Cl}$ : 4.1-7.0) were independently associated with being 'misclassified' in NNDRS.

Conclusions: We identified discrepancies in the accuracy of HBV case-reporting in the project hospitals. Onsite training on the use of anti-HBc IgM testing as well as on HBV case definitions and reporting procedures are needed to accurately assess program effectiveness and ensure case-patients are referred to appropriate treatment and care. Routine surveillance evaluations such as this can be useful for improving data quality and monitoring program effectiveness.
\end{abstract}

Keywords: Hepatitis B; acute hepatitis infection, Chronic hepatitis infection, Surveillance, Case-reporting

\footnotetext{
* Correspondence: nip2019@126.com; nip2019@126.com

${ }^{1}$ National Immunization Program, Chinese Center for Disease Control and Prevention, Beijing, China

Full list of author information is available at the end of the article
}

(c) The Author(s). 2020 Open Access This article is licensed under a Creative Commons Attribution 4.0 International License, which permits use, sharing, adaptation, distribution and reproduction in any medium or format, as long as you give appropriate credit to the original author(s) and the source, provide a link to the Creative Commons licence, and indicate if changes were made. The images or other third party material in this article are included in the article's Creative Commons licence, unless indicated otherwise in a credit line to the material. If material is not included in the article's Creative Commons licence and your intended use is not permitted by statutory regulation or exceeds the permitted use, you will need to obtain permission directly from the copyright holder. To view a copy of this licence, visit http://creativecommons.org/licenses/by/4.0/ The Creative Commons Public Domain Dedication waiver (http://creativecommons.org/publicdomain/zero/1.0/) applies to the data made available in this article, unless otherwise stated in a credit line to the data. 


\section{Background}

Globally, 257 million persons have chronic hepatitis B virus infections (HBV) and nearly 900,000 HBV-related deaths occur annually [1]. To address this disease burden, the World Health Organization (WHO) outlined a new strategy for viral hepatitis elimination, focusing on a $90 \%$ reduction of new chronic viral hepatitis B cases by 2030 [2]. China has reduced HBV transmission in persons born after 1992 through the successful implementation of a HBV vaccination program [3, 4]. By 2010, more than $98 \%$ of children were completing the three-dose hepatitis B vaccination series each year [5]. However, an estimated 90 million individuals of whom the majority are older than 30 years of age [6], are HBV surface antigen positive (HBsAg+) and at risk of developing cirrhosis and liver cancer $[7,8]$. Persons who are HBsAg+ can transmit HBV to susceptible persons.

Approximately $10 \%$ of the population in China was identified as HBsAg+ in a 1992 sero-survey [9]. To monitor changes in the prevalence of $\mathrm{HBsAg}+$, the $\mathrm{Na}$ tional Health Commission (NHC) (formerly the National Health and Family Planning Commission) implemented a policy requiring hospital staff to report all newly identified HBsAg + case-patients to the National Notifiable Disease Reporting System (NNDRS). NNDRS is a passive web-based surveillance system that relies on clinicians to report HBV infections as either acute, chronic or nonclassifiable HBV infections, based on the case definitions outlined in the national HBV reporting guidelines [10]. The system is available in all hospitals in China and can be used to monitor the accuracy and occurrence of acute and chronic HBV case reports. Passive surveillance is generally less costly to implement and maintain than active surveillance. Relying on clinicians for case reporting, however, can negatively affect the accuracy of the surveillance data, particularly if interpretations of the case definitions and diagnostic criteria are highly variable [11-13]. Previous evaluations of acute HBV casereporting to NNDRS in Yunnan, Shanghai, Tianjin, and Qinghai, for example, indicated that only 4-37\% of acute cases were reported correctly, according to the national case definitions [14-18]. These findings can affect the validity of $\mathrm{HBV}$ incidence estimates, the timely identification of HBV outbreaks, and the ability to appropriately target HBV prevention and control interventions.

In this project, we evaluated the accuracy of HBV surveillance data reported to NNDRS from hospitals in three geographically and demographically diverse provinces and identified factors that may affect the accuracy of these reports. We anticipate that the findings from this project can be used to strengthen China's HBV surveillance program to monitor the occurrence of acute and chronic infections and to assist it in achieving its global hepatitis B elimination goals [2]. When combined with more resource intensive sero-surveys [19, 20], the methods described in this paper could also be adapted and implemented in other high HBV burden countries as a supplemental tool for monitoring hepatitis B program effectiveness.

\section{Methods}

\section{Project site selection}

We evaluated HBV case-reporting in Fujian, Hainan, and Gansu Provinces (Fig. 1). Fujian is located on the eastern coast and has a population of 37 million; Gansu is located in the west and has a population of 26 million; and Hainan, is the smallest province and an island, with a population of approximately 9 million in 2010 [21]. The estimated population prevalence of $\mathrm{HBsAg}+$ is $4.4 \%$ in Gansu, $11.9 \%$ in Hainan, and $15.5 \%$ in Fujian [22].

In China, NHC rates hospitals from grade 1 to 3 based on the level of service availability. Grade 3 hospitals offer the highest comprehensive level of service. In each province, we selected one grade 2 and one grade 3 hospitals for a total of six project hospitals using the following criteria: 1) the hospital had an advanced laboratory information system (LIS) with access to HBsAg test results; 2) the hospital had an electronic hospital information system (HIS) that can link the LIS to the inpatient medical record number; and 3) in 2015, the hospital reported a greater number of hepatitis B cases compared to the hospital-based provincial mean. Additionally, administrators at each hospital agreed to participate in the project. Because more than $90 \%$ of hepatitis B cases are reported from grade 2 and 3 hospitals, we elected not to include other types of health care facilities in this project.

\section{Case inclusion criteria and data collection}

We included hepatitis B cases from the six participating hospitals that were reported to NNDRS from 1 January to 31 December 2015 and had a LIS record indicating $\mathrm{HBsAg}+$ test result for the same time-period. We downloaded all HBV cases that were reported to NNDRS from the six hospitals and linked these case reports to LIS data by name, gender, and birthdate. NNDRS records that could not be linked to LIS data and outpatients without record numbers were excluded from the analysis. We developed a standard abstraction form to collect medical record data from the six project hospitals for the reported HBV cases that were successfully linked to LIS. Staff from the Chinese Center for Disease Control and Prevention (China CDC) and the six hospitals used the form to collect the following information: reason for admission, admission ward (HBV-related wards included internal medicine, infectious disease, gastroenterology, and liver disease; non-HBV-related wards included surgery, pediatrics, obstetrics and gynecology, 


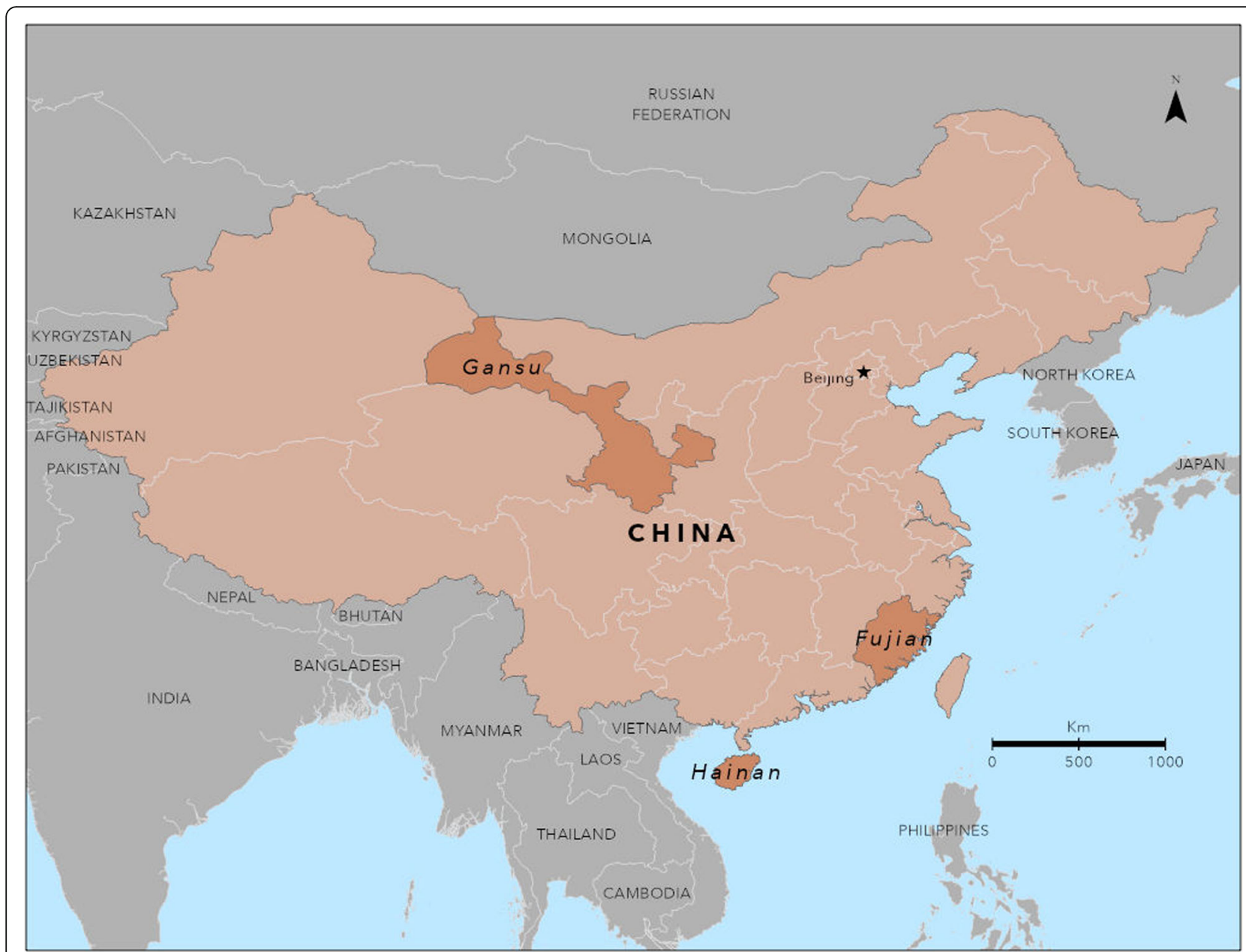

Fig. 1 Location of Fujian, Hainan, and Gansu Provinces participating in the hepatitis B surveillance project, China 2015

pulmonology, endocrinology, nephrology, orthopedics, neurosurgery, and traditional Chinese medicine), discharge diagnoses, clinical information including signs and symptoms of viral hepatitis, liver function tests, HBV DNA viral load, and hepatitis B sero-markers including HBV core antibody IgM (anti-HBc IgM), IgM antibody for hepatitis A virus (anti-HAV IgM) and hepatitis E virus (anti-HEV IgM), and hepatitis C virus antibody (anti-HCV). We obtained information on a patient's classification as an acute or chronic hepatitis B case from the hospital's information system.

\section{Data management and analysis}

We double-entered and verified data abstracted from the medical record in EpiData (version 3.1, Denmark). The resulting EpiData database was merged with the NNDR S-LIS case data using the patient medical record number and imported into SPSS (version 23, IBM, New York, USA) for analysis. We generated descriptive statistics on HBV cases by province, hospital grade, ward type, and patient characteristics. We compared the classification of hepatitis cases reported to NNDRS with the national HBV case definitions.

According to the national case definitions, hepatitis B patients should be classified as follows:

(1) HBV carriers: HBsAg-positive patient with no signs or symptoms of liver disease and normal alanine aminotransferase (ALT) levels ( $\leq 40 \mathrm{IU} / \mathrm{mL}$ ).

(2) Acute HBV: HBsAg-positive patient, who has signs or symptoms of liver disease or abnormal ALT levels $(>40 \mathrm{IU} / \mathrm{mL})$ without chronic inflammatory changes reported on abdominal ultrasound and negative hepatitis $\mathrm{A}$ and hepatitis $\mathrm{E}$ serologies, and who has clear evidence of HBV infection for less than 6 months or anti-HBc IgM positive.

(3) Chronic HBV: HBsAg-positive patient, who has signs or symptoms of chronic liver disease or abnormal ALT levels ( $>40 \mathrm{IU} / \mathrm{mL}$ ), and with at least one of the following: previous history of HBV infection $\geq 6$ months before, chronic inflammatory 
changes reported on abdominal ultrasound, or anti$\mathrm{HBC}$ IgM negative.

(4) Cirrhosis: HBsAg-positive patient with liver cirrhosis as reported via abdominal ultrasound, computerized tomography $(\mathrm{CT})$, or magnetic resonance imaging (MRI).

(5) Hepatocellular carcinoma (HCC): patient with liver lesion(s) that are suggestive of $\mathrm{HCC}$ reported via abdominal ultrasound, CT, or MRI together with alpha fetoprotein (AFP) $>400 \mu \mathrm{g} / \mathrm{mL}$.

(6) Non-classifiable $H B V$ : HBsAg-positive patient with only signs and symptoms of liver disease and no additional information, or an HBsAg-positive patient with insufficient information for classification.

We generated descriptive statistics on the clinical characteristics and laboratory test results from HBV patients who were included in the final database. Patients who were reported to NNDRS as having acute HBV infection were considered to be correctly classified if they met the case definition for acute HBV based on the medical record review. Similarly, patients reported to NNDRS as having chronic HBV were considered to be correctly classified if they met the case definition of chronic HBV, cirrhosis, or $\mathrm{HCC}$, based on the medical record review. The remaining patients were considered misclassified, or unable to be classified because of missing laboratory data. We calculated the percentage of patients who were correctly and incorrectly classified by province, hospital grade, gender, age, ward type, and primary discharge diagnosis (HBV or other). We stratified this analysis by NNDRS reporting status (i.e., reported as an acute or chronic HBV patient). In this bivariate analysis, all hospital and patient characteristics, except for province (three categories) and age (four categories), were dichotomized. An alpha level of 0.05 was applied to assess statistical significance.

Using classification status-NDRSS case-reports that were correctly classified and case-reports that were misclassified (including unable to classify) - as our binary dependent variable, we developed a logistic regression model to estimate the crude association of each of the above characteristics (e.g., province, hospital grade, and ward type) with an incorrect HBV case classification. Characteristics that were statistically significant in the crude analysis were included in our multivariable logistic regression model. Unadjusted and adjusted odds ratios (OR) and 95\% confidence intervals $(C I)$ were calculated. Finally, using available medical record information, we described the characteristics of HBV case-reports that were misclassified as acute and chronic in NNDRS.

\section{Ethics approval}

The China CDC Ethics Review Committee approved the project as a program evaluation. The United States
Centers for Disease Control and Prevention approved the project as a routine surveillance activity. We maintained all project-related data on a secure and passwordprotected computer.

\section{Results}

From 1 January-31 December 2015, 2064 HBV patients were reported to NNDRS by the six participating hospitals. Of these, 644 (31.2\%) were excluded from the analysis: 236 did not have HBsAg+ test results in LIS and 408 were outpatients (Fig. 2). Among the remaining 1420 patients, 881 (62.0\%) were from Fujian Province, $267(18.8 \%)$ were from Hainan, and $272(19.2 \%)$ were from Gansu. Most patients $(n=1014,71.4 \%)$ were reported from grade 3 hospitals, were male $(n=991$, $69.8 \%)$, and were $\geq 40$ years of age $(n=873,61.5 \%)$.

The percentage of cases reported to NNDRS as chronic HBV was $12.5 \%$ (34/272) in Gansu Province, $61.8 \%(165 / 267)$ in Hainan, and $98.6 \%(869 / 881)$ in $\mathrm{Fu}-$ jian. The percentage of cases reported to NNDRS as acute HBV was $87.5 \%(238 / 272)$ in Gansu Province, $38.2 \%(102 / 267)$ in Hainan, and 1.4\% (12/881) in Fujian. Across all project hospitals, the majority (57.7\%) of HBV cases were reported from HBV-related wards (Table 1). A larger percentage of patients from HBV-related departments were assessed for signs and symptoms of HBV infection and underwent an abdominal ultrasound or testing for HBV DNA, hepatitis A, C, and E compared to patients from non-HBV-related wards (data not shown, all $p$-values $<0.05$ ). Regardless of the ward, only $1.4 \%$ of all patients underwent anti-HBc IgM testing. Of the 352 reported acute HBV patients, only 34.4, 0.3, and $2.8 \%$ of patients received testing for anti-HAV IgM, antiHEV IgM, and anti-HBc IgM, respectively. Less than half of all HBV patients had medical record documentation of a hepatitis $B$ diagnosis.

Of the 352 patients reported to NNDRS as having acute HBV infections, 23 (6.5\%) were correctly classified as having acute HBV. The remaining 329 included 45 (12.8\%) patients who should have been reported as HBV carriers, $100(28.4 \%)$ as chronic HBV cases, $17(4.8 \%)$ as cirrhotic cases, 11 (3.1\%) with HCC, and 156 (44.3\%) who were non-classifiable (i.e., missing diagnostic information) (Fig. 3). Of the 1068 patients who were reported to NNDRS as having a chronic infection, 648 (60.7\%) were correctly classified in NNDRS at the time of the initial report. The remaining 420 included 143 (13.4\%) patients who should have been reported as HBV carriers, two $(0.2 \%)$ as having an acute HBV infection, and 275 (25.7\%) who were considered to be non-classifiable. In our bivariate analysis of acute $\mathrm{HBV}$ case-reports to NNDRS, province, hospital grade, ward type (HBV or non-HBV related), and having a discharge diagnosis of $\mathrm{HBV}$ were statistically associated with an incorrect HBV 


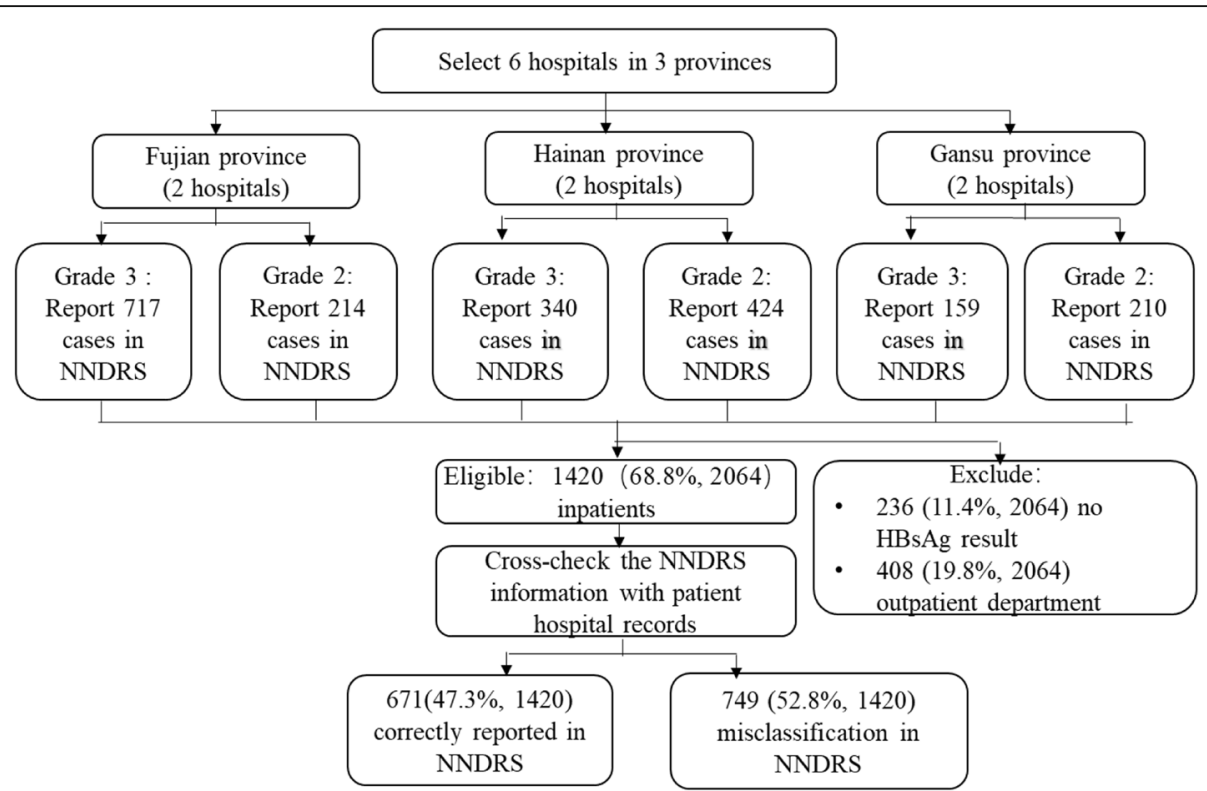

Fig. 2 Identification of eligible cases for review from six project hospitals in Fujian, Hainan, Gansu Provinces, China, 2015

Table 1 Demographic and clinical information on hepatitis B patients reported to the National Notifiable Disease Reporting System (NNDRS) from six project hospitals in Fujian, Hainan and Gansu Provinces, China, 2015

\begin{tabular}{|c|c|c|c|c|c|c|c|c|}
\hline & \multicolumn{2}{|c|}{ Fujian $(\boldsymbol{N}=881)$} & \multicolumn{2}{|c|}{ Hainan $(\boldsymbol{N}=267)$} & \multicolumn{2}{|c|}{ Gansu ( $\boldsymbol{N}=272)$} & \multicolumn{2}{|c|}{ Total $(\boldsymbol{N}=1420)$} \\
\hline & $\mathrm{N}$ & $\%$ & $n$ & $\%$ & $\mathrm{~N}$ & $\%$ & $\mathrm{~N}$ & $\%$ \\
\hline \multicolumn{9}{|l|}{ Hospital Grade } \\
\hline 2 & 191 & 21.7 & 95 & 35.6 & 120 & 44.1 & 406 & 28.6 \\
\hline 3 & 690 & 78.3 & 172 & 64.4 & 152 & 55.9 & 1014 & 71.4 \\
\hline \multicolumn{9}{|l|}{ Gender } \\
\hline Male & 632 & 71.7 & 189 & 70.8 & 170 & 62.5 & 991 & 69.8 \\
\hline Female & 249 & 28.3 & 78 & 29.2 & 102 & 37.5 & 429 & 30.2 \\
\hline \multicolumn{9}{|l|}{ Age group(year) } \\
\hline $0-24$ & 64 & 7.3 & 38 & 14.2 & 28 & 10.3 & 130 & 9.2 \\
\hline $25-39$ & 283 & 32.1 & 84 & 31.5 & 50 & 18.4 & 417 & 29.4 \\
\hline $40-59$ & 384 & 43.6 & 95 & 35.6 & 131 & 48.2 & 610 & 43.0 \\
\hline$\geq 60$ & 150 & 17.0 & 50 & 18.7 & 63 & 23.2 & 263 & 18.5 \\
\hline \multicolumn{9}{|l|}{ Report in NNDRS } \\
\hline Acute hepatitis B & 12 & 1.4 & 102 & 38.2 & 238 & 87.5 & 352 & 24.8 \\
\hline Chronic hepatitis B & 869 & 98.6 & 165 & 61.8 & 34 & 12.5 & 1068 & 75.2 \\
\hline \multicolumn{9}{|c|}{ HBV-related departments* } \\
\hline Yes & 612 & 69.5 & 126 & 47.2 & 81 & 29.8 & 819 & 57.7 \\
\hline No & 269 & 30.5 & 141 & 52.8 & 191 & 70.2 & 601 & 42.3 \\
\hline \multicolumn{9}{|c|}{ Primary discharge diagnosis } \\
\hline Yes & 385 & 43.7 & 91 & 34.1 & 36 & 13.2 & 512 & 36.1 \\
\hline No & 496 & 56.3 & 176 & 65.9 & 236 & 86.8 & 908 & 63.9 \\
\hline \multicolumn{9}{|c|}{ Specified acute or chronic diagnosis by clinicians } \\
\hline Yes & 429 & 48.7 & 84 & 31.5 & 81 & 29.8 & 594 & 41.8 \\
\hline No & 452 & 51.3 & 183 & 68.5 & 191 & 70.2 & 826 & 58.2 \\
\hline
\end{tabular}

*HBV - hepatitis B virus 


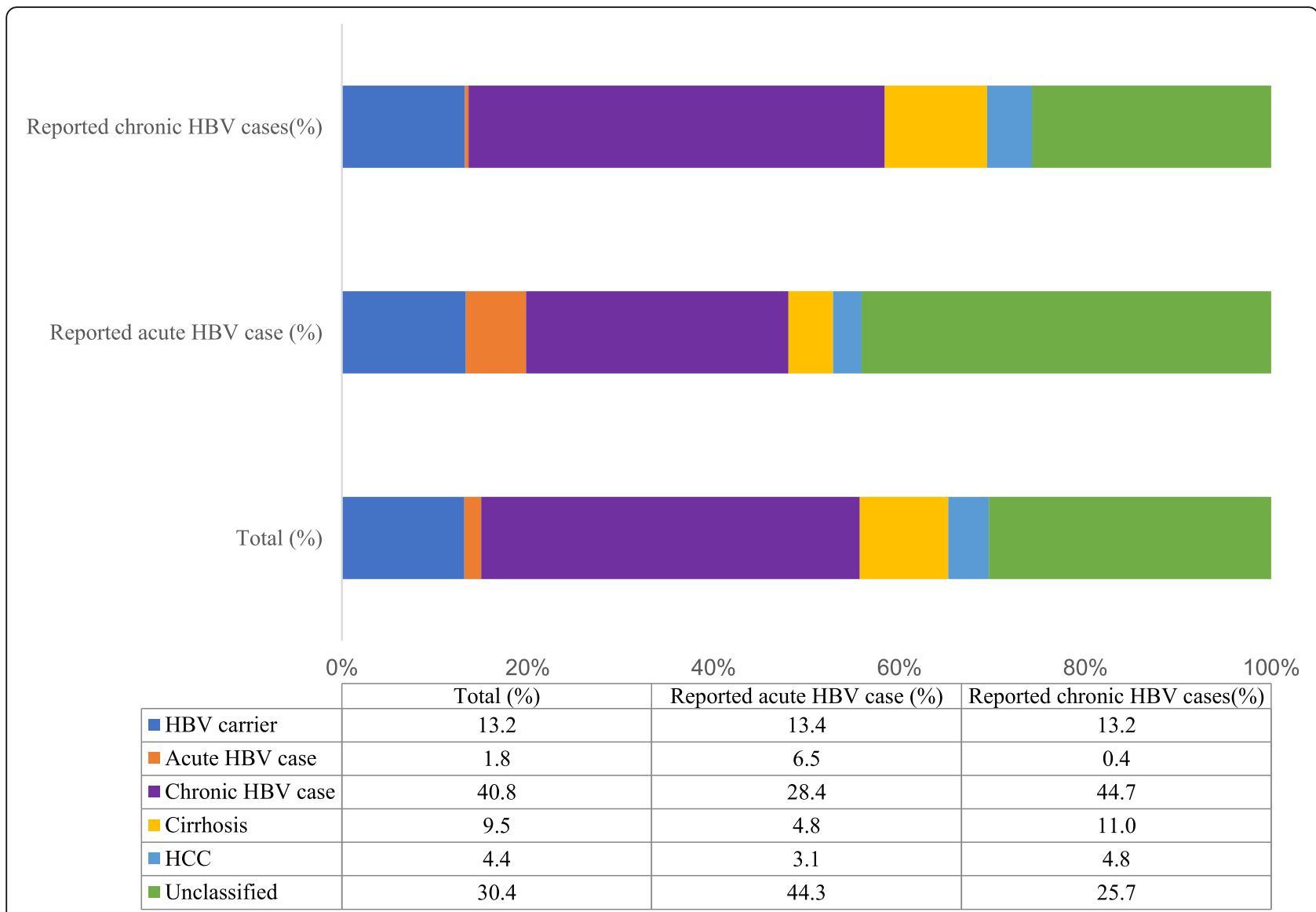

Fig. 3 Corrected case-classification of Hepatitis B cases reported to the National Notifiable Disease Reporting System from six project hospitals based on information abstracted from patients' medical records, Fujian, Hainan and Gansu Provinces, China, 2015

case-classification (all $p$-values $<0.05$; Table 2). Of the chronic HBV case-reports to NNDRS, gender, age, ward type, and having a discharge diagnosis of HBV were statistically associated with an incorrect caseclassification (all $p$-values <0.05). Overall, $25(1.8 \%)$ of the reported HBV patients could be considered to have acute infections based on the available information. Of these, 19 (76\%) were from Hainan Province, and the remaining five $(20 \%)$ were less than 24 years of age and eligible to have received the HBV vaccination as children.

When we combined all correctly classified and misclassified case-reports in our crude analysis, province, hospital grade, ward type, female, and older patients were statistically associated with having an incorrect case-classification (all p-values $<0.05$; Table 3 ). In the multivariable analysis, misclassified case-reports were more likely to be from Gansu (aOR $=12.7,95 \%$ CI:7.720.1) and Hainan (aOR $=1.8,95 \%$ CI:1.3-2.4), admitted to a grade 2 hospital (aOR $=1.6,95 \%$ CI:1.2-2.2), and from a non-HBV-related wards $(\mathrm{aOR}=5.3$, 95\% CI:4.17.0). Neither age nor gender were statistically significant in the multivariable model.
The major reason identified for incorrectly reporting patients as having an acute $(n=120,36.5 \%)$ or chronic ( $n=207,49.3 \%)$ infection was lack of additional history or diagnostic testing accompanying abnormal ALT results. The lack of testing following identification of abnormal signs and symptoms as well as ALT test results were also related to incorrect acute $(n=36,10.9 \%)$ and chronic reporting $(n=68,16.2 \%)$. Additionally, 41 (12.5\%) of the misclassified acute HBV infections and 15 (3.6\%) of misclassified chronic HBV infections had discharge diagnoses that differed from the corresponding NNDRS case classification (Table 4).

\section{Discussion}

Our evaluation identified discrepancies in the classification of HBV cases reported to NNDRS-only $60 \%$ of chronic HBV cases and 7\% of acute HBV cases were reported correctly to NNDRS. Most misclassifications involved patients with chronic HBV or HBV carriers or patients without the required diagnostic information who were reported as having an acute infection. Misclassification varied by province, hospital grade, and ward type. Additionally, only a few patients received 
Table 2 Hepatitis B cases reported to the National Notifiable Disease Reporting System by original and revised* case classification from six project hospitals in Fujian, Hainan, and Gansu Provinces, China, 2015

\begin{tabular}{|c|c|c|c|c|c|c|c|c|c|}
\hline & Cases & Acute Hep & is $B$ & & & Chronic $\mathrm{He}$ & titis B & & \\
\hline & & Reported & Revised & $\%$ Accurate & $\overline{x^{2}, \boldsymbol{p} \text {-value }}$ & Reported & Revised & $\%$ Accurate & $\overline{x^{2}, \boldsymbol{p} \text {-value }}$ \\
\hline Province & & & & & $<0.001$ & & & & 0.18 \\
\hline Fujian & 881 & 12 & 1 & 8.3 & & 869 & 538 & 61.9 & \\
\hline Hainan & 267 & 102 & 17 & 16.7 & & 165 & 93 & 56.4 & \\
\hline Gansu & 272 & 238 & 5 & 2.10 & & 34 & 17 & 50.0 & \\
\hline Hospital Grade & & & & & 0.004 & & & & 0.41 \\
\hline 2 & 406 & 223 & 21 & 9.4 & & 183 & 106 & 57.9 & \\
\hline 3 & 1014 & 129 & 2 & 1.6 & & 885 & 542 & 61.3 & \\
\hline Gender & & & & & 1.0 & & & & $<0.001$ \\
\hline Male & 991 & 218 & 14 & 6.4 & & 773 & 498 & 64.4 & \\
\hline Female & 429 & 134 & 9 & 6.7 & & 295 & 150 & 50.9 & \\
\hline Age group(year) & & & & & $0.33^{\#}$ & & & & 0.007 \\
\hline $0-24$ & 130 & 45 & 5 & 11.1 & & 85 & 60 & 70.6 & \\
\hline $25-39$ & 417 & 91 & 6 & 6.6 & & 326 & 206 & 63.2 & \\
\hline $40-59$ & 610 & 144 & 10 & 6.9 & & 466 & 285 & 61.2 & \\
\hline$\geq 60$ & 263 & 72 & 2 & 2.8 & & 191 & 97 & 50.8 & \\
\hline HBV related depa & ments & & & & $<0.001$ & & & & $<0.001$ \\
\hline Yes & 819 & 120 & 17 & 14.2 & & 699 & 530 & 75.8 & \\
\hline No & 601 & 232 & 6 & 2.6 & & 369 & 118 & 32.0 & \\
\hline Primary discharge & diagnosis & & & & $<0.001$ & & & & $<0.001$ \\
\hline Yes & 512 & 62 & 18 & 29.0 & & 450 & 438 & 97.3 & \\
\hline No & 908 & 290 & 5 & 1.7 & & 618 & 210 & 34.0 & \\
\hline Total & 1420 & 352 & 23 & 6.5 & & 1068 & 648 & 60.7 & \\
\hline
\end{tabular}

*Revised case-classification based on information abstracted from patients' medical record

\# Fisher's exact tests

anti-HBc IgM laboratory tests for differentiating acute and chronic infections as recommended. These discrepancies can be addressed through training, possible revisions to reporting procedures, and development of feedback mechanisms to hospital staff. Our project highlights the importance of routine surveillance evaluations for assessing data quality and monitoring program effectiveness.

Correct case classification in our study was substantially lower than observed in previous evaluations in other locations in China, including in Chongqing, Shandong, Hunan, and Zhejiang Provinces [23-26]. These evaluations suggested that between 63 and $84 \%$ of all diagnosed HBV patients were correctly reported to NNDR $\mathrm{S}$. This difference could reflect inconsistency in hepatitis B reporting practices across provinces [27] as well as variability in the methodologies applied in these surveillance evaluations. The WHO has recently prioritized standardized indicator-based surveillance and monitoring tools in the global viral hepatitis elimination strategy that could be used to ensure comparability of the results
[2]. Despite these differences, the correct HBV case classification was consistency lower in grade 2 hospitals for all evaluations conducted in China. Because health care staff at grade 3 hospitals in China typically have advanced degrees in medicine and research, these findings could suggest differences in training and clinical awareness. Lack of awareness among health care workers and laboratory staff was also identified as a major gap in viral hepatitis case detection and reporting in a survey on HBV and HCV testing and service delivery in low and middle-income countries [28]. Staffing constraints and doctor-to-patient ratios could also affect the correct case classification and reporting $[29,30]$. Targeting lowerlevel hospital staff with specific interventions, including site visits and regular reviews, may increase the reporting accuracy.

The accuracy of HBV case-reporting also varied by hospital ward. In Fujian, for example, nearly $70 \%$ of HBV cases were reported by a ward that was responsible for liver diseases. These patients were primarily seen for liver-related symptoms, resulting in more extensive use 
Table 3 Associations between hospital and demographic factors and Hepatitis B case classification to the National Notifiable Disease Reporting System, six project hospitals in Fujian, Hainan, and Gansu Provinces, China, 2015

\begin{tabular}{|c|c|c|c|c|c|c|c|}
\hline & \multirow{2}{*}{$\begin{array}{l}\text { Total } \\
\text { case-reports } \\
\mathrm{n}\end{array}$} & \multirow{2}{*}{$\begin{array}{l}\text { Misclassified } \\
\text { Cases } \\
\text { n (\%) }\end{array}$} & \multirow{2}{*}{$\begin{array}{l}\text { Correctly classified } \\
\text { cases } \\
\text { n (\%) }\end{array}$} & \multicolumn{2}{|c|}{$\begin{array}{l}\text { Crude analysis } \\
\text { for misclassification }\end{array}$} & \multicolumn{2}{|c|}{$\begin{array}{l}\text { Multivariable analysis } \\
\text { for misclassification }\end{array}$} \\
\hline & & & & OR $(95 \% \mathrm{Cl})$ & $p$-value & aOR $(95 \% \mathrm{Cl})$ & $p$-value \\
\hline \multicolumn{8}{|l|}{ Province } \\
\hline Fujian & 881 & $342(45.7 \%)$ & $539(80.3 \%)$ & referent & $<0.05$ & reference & $<0.05$ \\
\hline Hainan & 267 & $157(21.0 \%)$ & $110(16.4 \%)$ & $2.4(1.8-3.2)$ & & $1.8(1.3-2.4)$ & \\
\hline Gansu & 272 & $250(33.4 \%)$ & $22(3.3 \%)$ & $18.8(11.8-30.0)$ & & $12.7(7.7-20.1)$ & \\
\hline \multicolumn{8}{|c|}{ Hospital Grade } \\
\hline 2 & 406 & $279(37.2 \%)$ & $127(18.9 \%)$ & $2.6(2.0-3.3)$ & $<0.05$ & $1.6(1.2-2.2)$ & $<0.05$ \\
\hline 3 & 1014 & $470(62.8 \%)$ & $544(81.1 \%)$ & reference & & reference & \\
\hline \multicolumn{8}{|l|}{ Gender } \\
\hline Male & 991 & $479(64.0 \%)$ & $512(76.3 \%)$ & reference & $<0.05$ & reference & 0.06 \\
\hline Female & 429 & $270(36.0 \%)$ & $159(23.7 \%)$ & $1.8(1.5-2.3)$ & & $1.3(0.9-1.7)$ & \\
\hline \multicolumn{8}{|c|}{ Age group (years) } \\
\hline $0-24$ & 130 & $65(8.7 \%)$ & $65(9.7 \%)$ & $0.6(0.4-0.9)$ & $<0.05$ & $0.6(0.4-1.1)$ & 0.29 \\
\hline $25-39$ & 417 & 205 (27.4\%) & $212(31.6 \%)$ & $0.6(0.4-0.8)$ & & $0.8(0.5-1.2)$ & \\
\hline $40-59$ & 610 & $315(42.1 \%)$ & $295(44.0 \%)$ & $0.6(0.5-0.9)$ & & $0.7(0.5-1.1)$ & \\
\hline$\geq 60$ & 263 & 164 (21.9\%) & $99(14.8 \%)$ & reference & & reference & \\
\hline \multicolumn{8}{|c|}{ HBV related departments } \\
\hline Yes & 819 & $272(36.3 \%)$ & $547(81.5 \%)$ & reference & $<0.05$ & reference & $<0.05$ \\
\hline No & 601 & $477(63.7 \%)$ & $124(18.5 \%)$ & $7.8(6.1-9.9)$ & & $5.3(4.1-7.0)$ & \\
\hline Total & 1420 & 839 (100\%) & 671 (100\%) & - & - & - & - \\
\hline
\end{tabular}

of diagnostic testing and facilitating correct case reporting. Most hepatitis B patients ( $88 \%$ ) reported by non-liver disease wards-including surgery, pediatric, and gynecologic and obstetrics wards-at the two hospitals in Gansu Province may explain the number of patients who were misclassified in NNDRS. Hospitalized patients in China are routinely tested for $\mathrm{HBV}$ infection, and in order to help prevent mother-to-child transmission (PMTCT), the
NHC implemented a national policy requiring antenatal HBsAg testing for pregnant women in 2010 [31]. These strategies have increased HBV testing in non-liver disease wards. Strengthening the training on HBV case definitions for clinicians, especially for non-liver disease wards, could greatly improve the accuracy of HBV diagnosis.

Regardless of the ward, laboratory testing necessary for differential diagnosis was limited for the HBV patients

Table 4 Characteristics of 749 incorrectly classified Hepatitis B case reports from six project hospitals in Fujian, Hainan, and Gansu Provinces, China, 2015

\begin{tabular}{|c|c|c|c|}
\hline \multicolumn{2}{|l|}{ Characteristics } & \multirow{2}{*}{$\frac{\text { Numbers of cases }}{120}$} & \multirow{2}{*}{$\frac{\text { Percentage (\%) }}{36.5}$} \\
\hline Acute hepatitis case- report $(n=329)$ & ALT abnormal, no other information & & \\
\hline & Ultrasound abnormalities or history of HBV & 87 & 26.4 \\
\hline & No HBV history or clinical/ laboratory abnormalities & 45 & 13.7 \\
\hline & Diagnosed as chronic HBV & 41 & 12.5 \\
\hline & Abnormal signs/ symptoms and ALT only & 23 & 7.0 \\
\hline & Abnormal signs/symptoms only & 13 & 4.0 \\
\hline \multirow[t]{6}{*}{ Chronic hepatitis case-report $(n=420)$} & ALT abnormal, no other information & 207 & 49.3 \\
\hline & No clinical or diagnostic abnormalities (ultrasound performed) & 79 & 18.8 \\
\hline & No clinical or diagnostic abnormalities (ultrasound not performed) & 51 & 12.1 \\
\hline & Abnormal signs/symptoms and ALT only & 51 & 12.1 \\
\hline & Abnormal signs/symptoms only & 17 & 4.1 \\
\hline & Differs from clinical diagnosis & 15 & 3.6 \\
\hline
\end{tabular}


reported by the six participating hospitals. Only onethird of the reported acute HBV patients received testing for hepatitis A infections, and only 40 and $1.2 \%$ of patients seen in the liver disease wards were tested for anti-HEV IgM and anti-HBc IgM respectively. Frequency of testing for anti-HEV IgM and anti-Hbc IgM was even lower in non-liver disease wards. Because anti$\mathrm{HBc}$ IgM titers can be used to differentiate acute and chronic infections, WHO recommends anti-HBc IgM testing to assist with diagnosing recent infections, particularly if the patient's medical history or previous testing results are unknown. In our project, HBV patients were commonly misclassified as acute because of a lack of previous medical history and laboratory testing. Most of these patients would have likely been correctly classified as having chronic HBV by anti-HBc IgM testing if it had been performed as recommended [14]. Insufficient testing due to lack of clinical awareness or cost of testing was identified as a gap in generating quality epidemiologic data on hepatitis B infections in high burden countries [32].

Our findings may also reflect the current NNDRS reporting procedures. Testing hospitalized patients in China for HBsAg is routine practice [33], and all clinicians are required to report hepatitis $\mathrm{B}$ cases to NNDRS within $24 \mathrm{~h}$ of the initial diagnosis. Although clinicians can revise NNDRS case reports after additional diagnostic information becomes available, there is currently no incentive to do so. These classification discrepancies could be minimized by training hospital staff to reclassify NNDRS case reports when the final discharge diagnosis has been recorded in the hospital information system. Additionally, mechanisms should be created to ensure that regular reviews (i.e., monthly) are conducted to assess classification of NNDRS HBV case-reports. Routine feedback from China CDC on findings from the national hepatitis B surveillance program could also help local clinicians improve patient diagnosis and reporting practices [34].

Surveillance data are used to generate regional, national, and global estimates of acute and chronic HBV infections. The misclassification errors identified in our evaluation, as well as in previous studies, suggest that the incidence and prevalence of acute HBV and chronic HBV calculated from NNDRS are likely inaccurate. If we apply our re-classification results to the national HBV case-data from 2015, for example, the number of acute infections may be overestimated by as much as 37,000 and the number of chronic infections could be underestimated by more than 300,000 . These inaccuracies result in major challenges in identifying HBV outbreaks, evaluating the effectiveness of current prevention measures, and in efficiently allocating health care resources [19]. The 17 correctly classified acute infections from the two hospitals in Hainan, for example, could suggest missed opportunities to vaccinate and should be further investigated.

Our evaluation had several limitations. First, we conducted the evaluation in six hospitals in three provinces, limiting the generalizability of the results. Because of differences in the service area and population size of the project hospitals, our results do not reflect the incidence or prevalence of hepatitis B in the local population. Second, we targeted grade 2 and 3 hospitals; surveillance quality in lower level healthcare facilities was not evaluated. Based on our findings, additional training and support for HBV case-reporting at these lower level facilities would likely also be beneficial. Third, almost $30 \%$ of HBV case-reports to NNDRS could not be classified because of missing clinical information or testing results in LIS. We were also unable to evaluate reporting classification of outpatients who did not receive a hospital record number. The accuracy of the case classification of these HBV case-reports currently remains unknown. New approaches are needed to ensure that all HBV patients are identified and correctly reported to NNDRS. Finally, we were not able to assess the extent of under-reporting, meaning those patients who were diagnosed with acute or chronic HBV infections but who were not reported to NNDRS.

\section{Conclusion}

Our surveillance evaluation at six hospitals in three provinces identified discrepancies in the classification of HBV case-reports to NNDRS. Onsite training on the use of anti-HBc IgM testing as well as on HBV case definitions and reporting procedures are needed to accurately assess program effectiveness and ensure that patients are referred for the appropriate treatment and care. Routine feedback mechanisms from the national hepatitis B surveillance program would also be beneficial. Because surveillance data are used to monitor program effectiveness and progress toward global viral hepatitis elimination goals, our findings support the use of similar evaluations in other locations as a possible complement to resourceintensive HBV sero-surveys.

\section{Abbreviations \\ HBV: Hepatitis B virus; WHO: World Health Organization; HBsAg+: HBV surface antigen positive; NHC : National Health Commission; NNDRS: National Notifiable Disease Reporting System; LIS: Laboratory information system; HIS: Hospital information system; China CDC: Chinese Center for Disease Control and Prevention; anti-HBC IgM: HBV core antibody lgM; anti-HAV IgM: Antibody lgM for hepatitis A virus; anti-HCV: Antibody for hepatitis C virus; CT: Computerized tomography; MRI: Magnetic Resonance Imaging; HCC: Hepatocellular carcinoma; OR: Odds ratios; Cl: Confidence intervals}

\section{Acknowledgements}

We thank Fujian, Gansu and Hainan provincial and prefecture staff who contributed to the field work and Dr. Huai Yang for her contributions in developing the initial project protocol. 


\section{Authors' contributions}

The project was conceived by ZH, WFZ, ZR, CH, YZD, ALM, JJR, WHQ and ZGM. The statistical analysis was conceptualized and conducted by ZH, AJM, $J J R$, WFZ, and YQL. The manuscript was drafted by ZH, AJM, and JJR, and critically revised by AJM and JJR. All authors have read and approved the final version of the manuscript.

\section{Funding}

This project was funded by China-U.S. Collaborative Program on Emerging and Re-emerging Infectious Diseases (5U2GGH000961-04), and the Chinese Ministry of Science and Technology Program for Important Infectious Diseases Control and Prevention (2017ZX10105015).

\section{Availability of data and materials}

The datasets generated and/or analyzed during the current study are not publicly available due to reasons of patient confidentiality but are available from the corresponding author on reasonable request.

\section{Ethics approval and consent to participate}

The China CDC Ethical Review Committee approved the project as a program evaluation. The United States Centers for Disease Control and Prevention approved the project as a routine surveillance activity. We maintained all project related data on a secure and password protected computer.

\section{Consent for publication}

Not applicable.

\section{Competing interests}

We do not have any known conflicts of interest associated with this publication. There is no significant financial support for this work that could have influenced its outcome.

\section{Author details}

${ }^{1}$ National Immunization Program, Chinese Center for Disease Control and Prevention, Beijing, China. ${ }^{2}$ Division of Viral Hepatitis, United States Centers for Disease Control and Prevention, Atlanta, GA, USA. ${ }^{3}$ Division of Global Health Protection, United States, Centers for Disease Control and Prevention, Atlanta, GA, USA. ${ }^{4}$ Emerging Infections Program, Chinese Center of Disease Control and Prevention, Beijing, China.

Received: 7 May 2019 Accepted: 16 July 2020

Published online: 25 July 2020

\section{References}

1. World Health Organization. Global Hepatitis Report. Geneva: World Health Organization. 2017:2017. Available at: https://www.who.int/hepatitis/ publications/global-hepatitis-report2017/en/

2. World Health Organization. Global hepatitis sector strategy on viral hepatitis 2016-2021. Geneva, Switzerland: World Health Organization; 2016. Available at: https://www.who.int/hepatitis/strategy2016-2021/portal/en/. Last accessed on 6 May 2019

3. Cui FQ, Shen LP, Li L, Wang HQ, Wang FZ, Bi SL, et al. Prevention of chronic hepatitis $B$ after 3 decades of escalating vaccination policy. China Emerg Infect Dis. 2017:23(5):765-72

4. Cui FQ, Liang XF, Gong XH, Chen YS, Wang FZ, Zheng $H$, et al. Preventing hepatitis $B$ though universal vaccination: reduction of inequalities through the GAVI China project. Vaccine. 2013;31(Suppl 9):J29-35.

5. Cui F, Luo $H$, Wang $F$, Zheng $H$, Gong $X$, Chen $Y$, et al. Evaluation of policies and practices to prevent mother to child transmission of hepatitis $B$ virus in China: results from China GAVI project final evaluation. Vaccine. 2013; 31(Suppl 9):J36-42.

6. Woodring J, Pastore R, Brink A, Ishikawa N, Takashima Y, Tohme RA. Progress toward hepatitis B control and elimination of mother-to-child transmission of hepatitis B virus - Western Pacific region, 2005-2017. MMWR. 2019:68(8):195-200.

7. Sarin SK, Kumar M, Lau GK, Abbas Z, Chan HL, Chen CJ, et al. Asian-Pacific clinical practice guidelines on the management of hepatitis B: a 2015 update. Hepatol Int. 2016;10(1):1-98.
8. Hou J, Wang G, Wang F, Cheng J, Ren H, Zhuang H, et al. Guideline of prevention and treatment for chronic hepatitis B (2015 update). J Clin Trans Hepatol. 2017;5(4):297-318.

9. Dai ZC, Qi GM. Viral hepatitis in China (part one): Seroepidemiological survey in Chinese population 1992-1995. Beijing: Scientific and Technical Documentation Press; 1997.

10. Ministry of Health. Regulations on the management of infectious disease information reports. 2006. Available at: http://www.nhc.gov.cn/wjw/zcjd/2013 04/afb22001fbbe44e380b6956154dd850a.shtml. Last accessed on 6 May 2019.

11. Wang LP, Guo Q, Zhang CX, Guo Y, Zhao ZX, Ma JQ, et al. National investigation and analysis of hepatitis B reporting quality in 2006. Chinese Journal of Disease Control and Prevention. 2009;13(1):69-71.

12. Shi GQ, Wang P, Zhang L, Dong W, Xiao ZK, Liu R, et al. Evaluation of the role of information integration in the surveillance of viral hepatitis $B$. Disease Surveillance. 2006:21(6):317-20.

13. Lu Y, Cui FQ, Wang XJ, Gong XH, Dong HJ, Hu YS, et al. Investigation on acute hepatitis $b$ laboratory testing capacity in 8 provinces medical institutions. Chin J Epidemiol. 2006;27(9):802.

14. Wang FZ, Cui FQ, Lu Y, Chen YS, Zheng H, Liang XF. Analysis on reported hepatitis $B$ cases on pilot surveillance in 18 counties of 8 provinces of China. Chinese Journal of Vaccine and Immunization. 2007;13(4):309-11.

15. Miao N, Zhang GM, Wang FZ, Zheng H, Sun XJ, Ma XJ, et al. Consistency analysis on acute hepatitis $B$ inpatients reported by hepatitis B surveillance pilot spots in six provinces of China. Chinese Journal of Epidemiology. 2017; 38(2):216-20.

16. Kong Y, Ding ZR, Pang YK, Luo M, Huang GF, Kang WY. Analysis on the epidemiological characteristics and accuracy of reported incidence of hepatitis B virus (HBV) infection in Yunnan Province. Chinese Journal of Vaccines and Immunization. 2013;19(2):154-8.

17. Li W, Ma HB, Zhao ZC. Analysis and countermeasures on the quality of hepatitis B reports by medical institutions in Xining. Modern Preventive Medicine. 2010;37(3):552-4.

18. Miao N, Wang FZ, Zhang LJ, Zheng H, Sun XJ, Wang F. Evaluation on hepatitis B surveillance models at surveillance pilot points in China, 20132015. Chinese Journal of Epidemiology. 2017;38(12):1645-8.

19. World Health Organization. Technical considerations and case definitions to improve surveillance for viral hepatitis: technical report. Geneva, Switzerland: World Health Organization; 2016. Available at: http://www.who.int/about/ licensing/copyright_form/index.html. Last accessed on 6 May 2019.

20. German RR, Lee LM, Horan JM, Milstein RL, Pertowski CA, Waller MN, et al. Updated guidelines for evaluating public health surveillance systems: recommendations from the Guidelines Working Group. MMWR. 2001; 50(RR13):1-35.

21. Population Census Office under the State Council, Department of Population and Employment Statistics National Bureau of Statistics. Tabulation on the 2010 population census of the People's Republic of China, 2012. Available at: http://www.stats.gov.cn/tisj/pcsj/rkpc/6rp/indexch. htm. Last accessed on 6 May 2019

22. Ministry of Health, Chinese Center for Disease Control and Prevention. The National Report of Hepatitis B Sero-survey in China. Beijing: People's Medical Publishing House, 2010.

23. Chen YH, Chen B, Shi SJ, Hong SR, Yang HY, Zheng JF. Diagnosis and reporting of hepatitis B in 3 surveillance counties in Quanzhou. Fujian Disease Surveillance. 2014;29(12):999-1002.

24. Miao N, Zhang GM, Zheng H, Wu ZH, Sun XJ, Wang F, et al. Analysis of the hepatitis B report data on pilot surveillance in 200 counties in China, 2013. Chin J Prev Me. 2015;49(9):766-70.

25. Lin D, Yu XH, Pan QJ, Chen Y, Ni QX, Zhang XH, et al. The diagnostic coincidence rate of hepatitis $b$ diagnosis in Wenzhou medical institution in 2011. Modern Practical Medicine. 2012;24(7):798-803.

26. Jiang $X Y$. The evaluation of epidemic reporting equality and analysis of epidemic characteristics on hepatitis B in Jiyang County [D]: Shandong University; 2014. Available at: http://1.202.226.196:8888/rwt/WANFANG/http/ M6YHPZLPM3RX635EMF4GCLUDN7XT6Z5P/details/detail.do? type= degree\&id $=Y 2594720$.

27. Wei-shen W, Jing C, Hai-yan H, Ying Z, Chao L. The impact of surveillance and hospital uniform criteria on hepatitis B diagnosis and report. Chinese Journal of Disease Control \& Prevention. 2015:19(2):174-6.

28. Childs L, Roesel S, Tohme RA. Status and progress of hepatitis B control through vaccination in the South-East Asia region, 1992-2015. Vaccine. 2018 36(1):6-14. 
29. Ishizaki A, Bouscaillou J, Luhmann N, Liu S, Chua R, Walsh N, et al. Survey of programmatic experiences and challenges in delivery of hepatitis $B$ and $C$ testing in low- and middle-income countries. BMC Infect Dis. 2017;17(Suppl 1):696.

30. Li X. The problems existing in the secondary general hospital human resource management and countermeasure. Chinese Journal of Primary Medicine and Pharmacy. 2018;25(14):1892-3.

31. Wang AL, Qiao YP, Wang LH, Fang LW, Wang F, Jin $X$, et al. Integrated prevention of mother-to-child transmission for human immunodeficiency virus, syphilis and hepatitis B virus in China. Bull World Health Organ. 2015; 93(1):52-6.

32. Wait S, Kell E, Hamid S, Muljono DH, Sollano J, Mohamed R, et al. Hepatitis B and hepatitis $C$ in southeast and southern Asia: challenges for governments. Lancet Gastroenterol Hepatol. 2016;1 (3):248-55.

33. Li XY, Xia G, Yuan PZ, Jiang YH, Gu C. Analysis of the results of China's standardized hepatitis B monitoring program in Neijiang. Chin J Gastroenterol Hepatol. 2017;26(2):203-6.

34. Richards CL, ladermarco MF, Atkinson D, Pinner RW, Yoon P, MacKenzie WR, et al. Advances in public health surveillance and information dissemination at the Centers for Disease Control and Prevention. Public Health Rep. 2017; 132(4):403-10

\section{Publisher's Note}

Springer Nature remains neutral with regard to jurisdictional claims in published maps and institutional affiliations.

Ready to submit your research? Choose BMC and benefit from:

- fast, convenient online submission

- thorough peer review by experienced researchers in your field

- rapid publication on acceptance

- support for research data, including large and complex data types

- gold Open Access which fosters wider collaboration and increased citations

- maximum visibility for your research: over $100 \mathrm{M}$ website views per year

At $\mathrm{BMC}$, research is always in progress.

Learn more biomedcentral.com/submissions 